\title{
Strangers in the House? Communication between Mothers and their Hearing Impaired Children who Sign
}

\author{
Lavanithum Joseph and Erna Alant
}

\author{
Centre for Augmentative and Alternative Communication \\ University of Pretoria
}

\begin{abstract}
Many hearing impaired children rely on signing as a method of communication and are educated through this medium at school. While there is a paucity of information on the use of signing in the home, the impression in the literature is that these children are often unable to communicate through this medium in their homes. This has serious implications for family relationships as well as the personal well - being, educational success and social integration of the child. The present study explored the signing experience of 45 mothers of children in the junior primary phase at schools for the deaf in the Durban region of Kwa-Zulu Natal as a reflection of the use of signing within the home. A descriptive survey design, using two researcher administered questionnaires, was used to obtain information on the signing practice of mothers, exploring aspects related to the extent to which signing is used, the type of signing used and signing proficiency. The findings revealed mothers' signing skill to be inadequate in terms of their own assessment on rating scales and descriptions of their signing. They signed less frequently than their children did, using speech more often, and with the vast majority using simultaneous communication when they did sign. Signing ability was judged to be below that of their children, with sign vocabularies of between $0-50$ words for $85 \%$ of the participants. It appears that sign learning by hearing parents of deaf children in this region needs to be actively promoted. The implications of these findings are discussed to address the communication needs of signing deaf children and their families within the South African context.
\end{abstract}

KEY WORDS: sign language, sign systems, signing proficiency, deafness, mothers of deaf children.

\section{INTRODUCTION}

The professions of 'Speech Language Therapy and Audiology in South Africa have concerned themselves with issues of signing for some time. Studies have been undertaken to assess the signing abilities of teachers (Aron et al., 1986) and resource development for sign learning (Cohen, 1996). Deaf education and Deaf culture in South Africa have been raised as pertinent issues (Penn and Reagan, 1991; Penn, 1993). More recently there have been - discussions around the issue of sign bilingualism in deaf education with reference to the profession (Joseph, 1998; Joseph, 2000; Noorbaai, 2000). Professional interest in the young deaf child begins during the period of diagnosis and early habilitation and interfaces with deaf education. The greatest task facing both parents and professionals working with deaf children in the early years is that of breaking the communication barrier that exists between a mother and her deaf child (Gregory, 1995).

Parents need to establish communication, as any withdrawal from interaction would later be played out in a complex way by society's withdrawal of communication from the deaf child (Hull, 1997). The decisions now facing parents, however, tends to be more complex because of the choice to sign or not to sign, and whether or not to stress signing with speech or sign language (Gregory, 1995). Parents are given options with regard to communication methods that are available, viz. oralism, total communication or bilingualism (Lynas, 1994), yet they often have to enter an education system that focuses on signing. For example, all eight public schools for the deaf in Kwa-Zulu Natal use signing as a medium of instruction. Thus parents have to sign out of necessity if they want to communicate with, and be part of their deaf child's world. The complexity of this situation cannot be underestimated. More than $90 \%$ of deaf children are born to hearing parents who do not know how to sign (Kyle and Woll, 1988). Acceptance of signing, learning to sign, and interacting meaningfully and easily with the child through signing is what is required.

The signing skills of parents, their attitude towards signing and the opportunities they have to learn to sign are strongly related to each other and have a direct bearing on their use of signing (Bornstein, 1990). In this respect there is a paucity of information on the experiences of mothers who sign. There are indications however, that signing is generally not exploited in the home environment. It appears that the communication methods used at school do not commonly match those used in the home, as speech rather than signing seems to dominate interaction among hearing parents and their signing deaf children (Bess and Humes, 1995). According to Bornstein (1990) half the children exposed to signing at school may not see it at home. The majority of deaf children learn to sign without input from their parents and $90 \%$ of parents are learning to sign at the 
same time as their child (Gregory and Hartley, 1991). Luterman (1987) states that most hearing parents do not develop sufficient signing skills to communicate fully with their children. It therefore appears that while there are parents who do learn and use signing to communicate with their deaf signing children, many do not.

Learning to sign requires an investment in time and effort that many parents are often unwilling and/or unable to make (Bornstein, 1990). Inadequate signing proficiency of parents of children who are in signing programmes in the education system can be attributed to barriers that parents encounter in their attempts to learn to sign. These can be broadly classified as distance from the learning centre, transport difficulties, time constraints due to employment and family responsibilities, language difficulties with regard to lack of proficiency in the spoken language of the school, lack of immersion in a signing environment, and the lack of resources (Swisher and Thompson, 1985; Cohen, 1996; Lynas, 1994).

Impoverished communication between signing children and their parents is evident in the numerous accounts of the difficulties, isolation and bewilderment that deaf children experience in situations that are considered normal in everyday life. These reports tell of children's inability to access information vital to their understanding of events around them, and of sharing their thoughts, joys and fears with those closest to them (Lane et al., 1996; Mindel and Vernon, 1987). In most instances, these are their parents and siblings. These children and their parents are described as being "strangers" in their own home (Mindel and Vernon, 1987:XIX). The terms being used to describe the failure to address the language needs of deaf children are "oppression" and "abuse" (Preston, 1994:127; DEAFSA, 1997:10). On the other hand, mothers of young deaf children have been reported as feeling "cutoff" from their children, often wondering what their children were thinking or feeling (Gregory, 1995:194). Mindel and Vernon (1987) show concern for the emotional frustrations that deaf children and their parents undergo and call for enhanced sympathy toward these families.

Information relating to parents' use of signing appears to be particularly relevant at this stage in deaf education. There have been significant changes in the status of sign language all over the world with important implications for deaf education and service provision. Sign language has been acknowledged as a legitimate language, equivalent to any spoken language (Kyle and Woll, 1988). Many researchers consider sign language as the first and natural language of Deaf people (Sacks, 1989; Stokoe, 1990). In keeping with these developments, sign language has been accepted by the South African constitution as a language that needs to be promoted, and the new South African Schools Act recognizes sign language as an official language for educational purposes (DEAFSA, 1997). Bilingualism as an approach in deaf education is being promoted by academics and the World Federation for the Deaf.

This educational philosophy has implications for the professions of Speech-Language Therapy and Audiology, These relate particularly to the issues of counselling at diagnosis, prescriptive practice, information dispensing following diagnosis, the role of the audiologist in parentinfant programmes and the role of speech training for the Deaf child. It has been stated that parents have not been served well by the professionals involved in the area of deafness (Mindel and Vernon, 1987) due to a lack of awareness of the communication needs of parents and their deaf children as well as a failure to provide them with realistic solutions. Related to this is the critical issue of lack of consensus among professionals in the field with regard to signing being a method of choice with the deaf child. Therefore, eliciting information from parents themselves about their signing experience, would appear to contribute to a better understanding of the unique needs of parents who need to sign. The extent to which signing is used within the home context by signing children and their parents would be explored in terms of meaningful communication that is perceived as mainly inadequate by most writers.

\section{METHODOLOGY}

\section{AIM}

The aim of the study was to describe the signing practice of mothers of junior primary school children in signing programmes at schools for the deaf in the Durban Metropolitan area.

\section{Objective:}

To describe mothers' perceptions of:

- Their use of signing in comparison to other methods of communication used

- The type of signing used

- Their signing proficiency

\section{RESEARCH DESIGN}

A descriptive survey design was employed with the use of a structured interview format. This method was considered suited to the data which were the perceptions of mothers (Leedy, 1993). The design, through the descriptive record allows scrutiny of the data as well as relations within the data, including the use of statistical techniques.

\section{SUBJECTS}

\section{Subject Selection Criteria}

The subjects needed to be mothers of children attending schools for the deaf where signing was used as a medium of instruction. Mothers were chosen as they are considered the main language stimulators in the home and the caregivers most likely to be involved in the intervention programme. In addition, mothers were considered to be more easily accessible and, by restricting the sample to mothers alone, the homogeneity of the sample would be increased. Legal guardians and primary care-givers such as grandmothers were included in keeping with the definition of parent used in the Public Discussion Document of the National Commission on Special Needs in Education and Training and the National Committee for Education Support Services (1997). Only schools in Durban registered with the Kwa-Zulu Natal Education Department were included. Children needed to be in the Junior Primary phase of education as the first level of formal schooling was seen as beneficial in terms of addressing the issue of parent-child communication in the early years of school life. Only children in grade one (entry 
level) and grade three (exit level) were selected as this was considered an adequate range of this phase. Thus the sampling method can be described as purposeful sampling.

\section{Description of schools}

All four public schools for the deaf in the Durban area were included in the study. These schools were based at Amanzimtoti, Gillits, Newlands West and Umlazi. Two schools were residential schools. All schools were English medium schools. The sign dialect varied amongst the schools, although all four schools embraced the concept of sign bilingualism.

\section{Description of mothers}

Forty five mothers participated in the study. Mothers came from a diversity of backgrounds.

Age: Most mothers (62\%) fell in the $30-39$ year age group.

Marital status and employment: A large number of mothers (39\%) were single mothers with $52 \%$ of mothers working.

Educational status: Most mothers (55\%) had not completed high school.

Language Background: More than a third of mothers (36\%) spoke Zulu in the home, while many of them (28\%) were not proficient in the language medium of the school, viz. English.

Residential background: The majority of mothers (91\%) lived in and around Durban. Home visits revealed that many mothers lived in poor conditions in both informal and formal dwellings, while some came from wealthy suburban homes.

\section{Description of children}

There were $42 \%$ males and $58 \%$ females in the study.

Age and grade: Children were between 5 and 13 years of age, with most of them (33\%) in the 8year category. Approximately half the children were in grade one (51\%) and half in grade two (49\%).

Hearing status: According to school records, hearing losses (based on the pure-tone average of the better ear unaided), fell within the range of moderate to profound. Most of the children (58\%) had profound losses, while 27\% had severe losses. Thus the majority of children (85\%) in the study could be classified as having severe and profound hearing impairment; with onset prelingually (89\%). Hearing aids were worn consistently (daily at home and at school) by a small percentage of these children (24\%), although $91 \%$ of them did wear hearing aids.

Signing background: All children were able to sign. Most children $(64 \%)$ had begun signing between the ages of 3 and 6 years with the majority signing for 3 years or more (78\%). The average number of years of signing was 4 years.

Spoken language: With reference to spoken language ability, $18 \%$ were described by their mothers as not using spoken language at all, while a further $58 \%$ were only able to communicate using a small vocabulary, speaking on a few topics. Thus $76 \%$ of the children were considered by their mothers to have inadequate spoken language ability.

Approximately a third of the children lived in the school residential facility.

\section{DESCRIPTION OF THE INSTRUMENT}

Two questionnaires, Questionnaire I-Mothers' Experience with Sign Communication: Background Information, and Questionnaire II-Mothers' Experience with Sign Communication: Methods of Communication were developed based on relevant literature and consultation with experts in the field with regard to the suitability of the questions in terms of structure and content.

Closed-ended questions using mainly multiple choice responses were used as it was felt to be an easier format because of the difficulties with terminology and concepts, and their implications for the analysis of responses and reliability of the instrument. Care was taken with the wording of questions so as to avoid ambiguity, maintain a logical sequence and provide an exhaustive range of possibilities with the category of "other" available to allow respondents freedom to express themselves.

The first questionnaire sought background information of both the mother and the child. The second questionnaire focussed specifically on issues relating to signing practice. Questionnaire I consisted of 20 questions that sought demographic information of mothers, mothers' communication background with regard to home language, language proficiency, and hearing status as well as identifying information of the children with regard to gender, age, grade and hearing status. Questionnaire II comprised of 19 questions that pertained to mothers' perceptions of their children's spoken language abilities, methods of communication (speech, natural gestures, signing, writing and 'other') used in parent-child communication in terms of frequency of use and the success of these modes in conveying information, mothers' rating of their own and their children's signing ability, and mothers' description of their signing with regard to range of topics, syntactical complexity and type of signing used. Rating scales were used to describe the use of signing in comparison to other methods of communication and to describe signing proficiency. The assessment of signing proficiency was based on the 5- point scale used by Metz et al. (1997) in the Language Background Questionnaire. According to the authors, the rating of signing skills on a scale of one to five, with one being equivalent to no skills and five being equivalent to excellent skills, was found to have reasonable criterion validity for self-assessment of sign language skills of adults as there was a high degree of congruence between self-assessed communication skills and formal measures of communication skills. The authors further cite the advantages of self -assessment instruments as including ease of administration, low expense, non-threatening and non-invasive nature, as well as validity of the tool in terms of face validity as self reports reflect personal experience. Mothers rated their signing proficiency both globally and specifically on a receptive level. Their global skill was taken as the main indicator of their signing skill. Information was also sought on the communication modes and signing proficiency of the children for purposes of comparison as parents have been observed to adjust their level of communication to suit that of their children (Gallaway and Richards, 1994).

Multiple-choice and multiple response questions were used to allow parents to describe the type of signing they used. A cross check was used to assess the validity of the response by probing with the use of the Centre for Assessment and Demographic Studies (CADS) measure 
to assess the type of signing used by teachers, based on the way teachers described how they would sign two English sentences (Woodward, 1990).

Both a pre-pilot and a pilot study were undertaken to test the instrument and assess the conditions under which the study would be carried out. Following these procedures adjustments were made to the wording of certain questions to improve clarity. The questionnaires were translated into Zulu as many children at the schools came from a Zuluspeaking background. The back-translation method was used.

Note: This article is based on an aspect of a study (Joseph, 1998) which may be consulted for a sample of the questions described above.

\section{PROCEDURE}

Mothers were accessed via the school. School principals granted permission for letters requesting parent participation to be sent home via the children selected for the study, viz. all children in grades one and grades three at the selected schools. Interviews were set up with all parents who indicated their willingness to participate in the study via return slips. Interviews were confirmed telephonically. The site of the interview was determined by parent preference such as the home, place of employment or school to encourage parent participation. Interviews were scheduled over a two- week period and were of approximately 20 minutes duration.

Questionnaires were administered via a structured interview by the researcher or a fieldworker who was fluent in Zulu and English. Fieldworkers were chosen for their familiarity with the interview process. Two fieldworkers were used. One fieldworker was a nurse at one of the schools and who had frequent contact with parents and often acted as an interpreter at the school. The other fieldworker was a student in her third year of study in Speech and Hearing Therapy and who had experience in interviewing parents and issues of communication. Both field workers received training in terms of administering the particular questionnaires via role-play and during the pilot study so as to ensure reliability and consistency across interviews. The interview was particularly important in obtaining data from those who were illiterate or who had low literacy levels and whose participation would be restricted by the use of a selfadministered questionnaire.

\section{DATA ANALYSIS}

The data was analysed using both descriptive and inferential statistics. Measures of central tendency (mean, median and mode) and measures of variability (standard deviation and range) were calculated for all variables in Questionnaire I and Questionnaire II. Correlation coefficients were calculated to determine the strength of the relationship between variables. A series of non-parametric tests including Chisquare, Spearman's Correlation Coefficient and Mann-Whitney tests were used in accordance with the nature of the data for which relationships were sought (Leedy, 1993).

\section{RESULTS AND DISCUSSION}

The signing practice of mothers is described in terms of three aspects, viz. their use of signing in comparison to other modes of communication used, the type of signing used, and their signing proficiency.

\section{USE OF SIGNING IN COMPARISON TO OTHER MODES OF COMMUNICATION}

The frequency of signing is presented both in terms of a comparison between its use with other modes of communication, and a comparison between its use by mothers and their children. An overview of communication modes used is presented in Table 1. Figure 1-3 describes the findings for specific modes.

\section{Use of signing}

Children tended to sign more frequently than their mothers did as more than half the sample (53\%) always used signing as a method of communication, compared to $40 \%$ of mothers who always used signing as a method, as is reflected in Figure 1. This is further exemplified by the mean responses on Table 1 which shows children as bordering on the category of "usually" using signing

TABLE 1: Frequency of use of the various modes of communication used by mothers and their children

(note: the 5 point rating scale used translated as $1=$ never, $2=$ rarely, $3=$ sometimes, $4=$ usually and $5=$ always).

\begin{tabular}{|l|c|c|c|c|}
\hline \multirow{2}{*}{$\begin{array}{l}\text { Communication } \\
\text { Mode }\end{array}$} & \multicolumn{2}{|c|}{ Mother } & \multicolumn{2}{c|}{ Child } \\
\cline { 2 - 5 } & $\overline{\mathbf{X}}$ & $\overline{\text { SD }}$ & $\overline{\mathbf{x}}$ & $\overline{\text { SD }}$ \\
\hline Signing & 3.60 & 1.44 & 3.89 & 1.35 \\
Speech & 3.69 & 1.51 & 3.42 & 1.57 \\
Gestures & 3.49 & 1.38 & 3.51 & 1.29 \\
Writing & 1.40 & 0.72 & 1.40 & 0.78 \\
Other & 1.46 & 0.87 & 1.36 & 0.81 \\
\hline
\end{tabular}

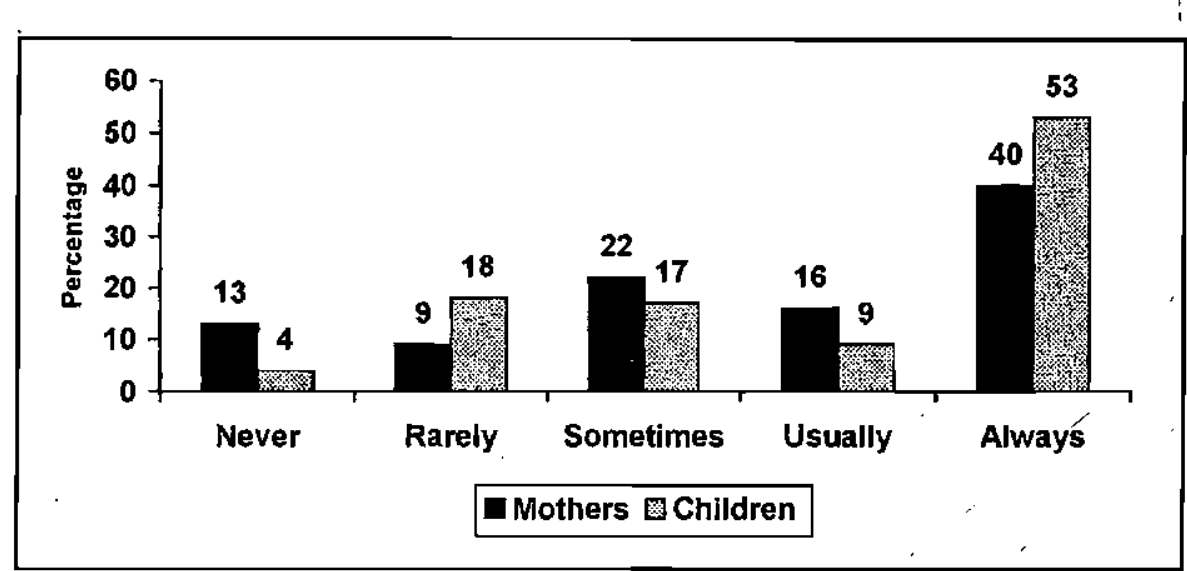

FIGURE 1: Comparison of mothers' and children's use of signing 
compared to mothers who were closer to the description of "sometimes" using signing. There was, however, a positive correlation $(\mathrm{r}=.7020, \mathrm{p}<.01)$ between mothers' and children's use of signing. Thus, as the child's tendency to sign increased so did their mother's signing. This tendency to match the child's mode of communication is reported by Gregory et al. (1995) in their study of deaf young people and their families. Parents whose children had been educated in the oral tradition had subsequently learned to sign or accompanied gesture with their speech if their children were using sign language in their later years.

\section{Use of speech}

There was a positive and significant relationship between mothers' use of speech and the child's use of speech $(\mathrm{r}=.6613, \mathrm{p}<.01)$. Once again parents and children tended to match the communication mode used. However, a comparison between mothers' use of signing and speech indicated a negative correlation $(r=-.4584, p<.01)$. It therefore appears that parents who were speaking tended not to sign or vice versa.

In addition, for those mothers who used speech to communicate, only about a third (31\%) found it always successful in conveying information to their child (results not presented). On the other hand, $22 \%$ of children who used speech were considered to be always successful in conveying information to their mothers. It therefore appears that speech was used by the majority of mothers despite it not always being successful. The use of speech by mothers of deaf children has been encouraged in both the oral and total communication approaches. Gregory (1995) reports that while many mothers in the oral tradition (33\% in her study) spoke constantly to their child whether they were listening or not, none reported not speaking at all. Preston (1994) confirms this by stating that even those deaf persons who were oral were no more likely to have good communication with hearing parents. Only $13 \%$ (37 of 288 ) of his sample of Deaf adults could communicate well with at least one parent.

\section{Use of gestures}

In terms of the relationship between mothers' use of gestures and signing, there was a positive correlation $(r=.5728, p<.01)$. This is unlike the relationship with mothers' use of speech and signing. It therefore appears that mothers who used formal signing more also tended to use gestures more. However, only $39 \%$ of mothers who used gestures found it always successful in conveying information. The need to use gestures seems to indicate a possible inadequacy of their formal signing skill as well as an inability to communicate through speech.

\section{Use of writing and other methods of communication}

Writing as a method of communication, was generally not exploited by both parents and children. The majority of parents and children (71\%) did not use writing at all as a method of communication. Those who did use it as a method tended to do so rarely. This could be considered a low result given that children were in the formal phase of schooling, with approximately half the children (49\%) in grade three where writing was being used in the educational programme. This is especially so given the difficulties that children, who used speech, were generally having in conveying and receiving information. Studies have shown that reading as a receptive mode is highly successful in communicating with deaf children (Grove, et al., 1984). The failure to explore writing has implications for the Bilingual approach which promotes the use of writing as a primary method of communication to be used in interaction. Gregory et al. (1995) also report parents' failure to use writing to communicate with young Deaf people. Only $1 \%(1$ in 82$)$ of parents reported using writing to communicate.

Similarly a large number of parents $(76 \%)$ did not use any other method to communicate. Those mothers who did use other methods, did so infrequently and used drawings $(11 \%)$, pictures $(7 \%)$, objects ( $4 \%$ ) and the "sign language" dictionary $(2 \%)$ to communicate. Over one third of the children $(38 \%)$ used other methods to communicate and these included the use of drawings (40\%), pictures $(4 \%)$ and the "sign language" dictionary (2\%). It appears therefore that there was a group of parents and children who felt the need to explore alternate means of communication as they could not communicate effectively with signing, gestures or speech. The fact that $40 \%$ of children

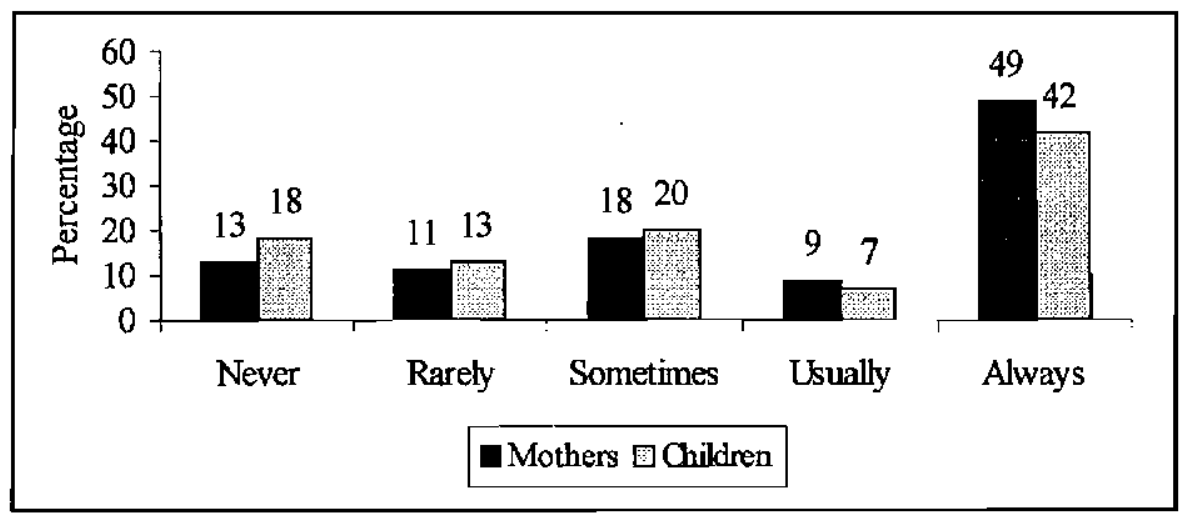

FIGURE 2: Comparison of mothers' and children's use of speech

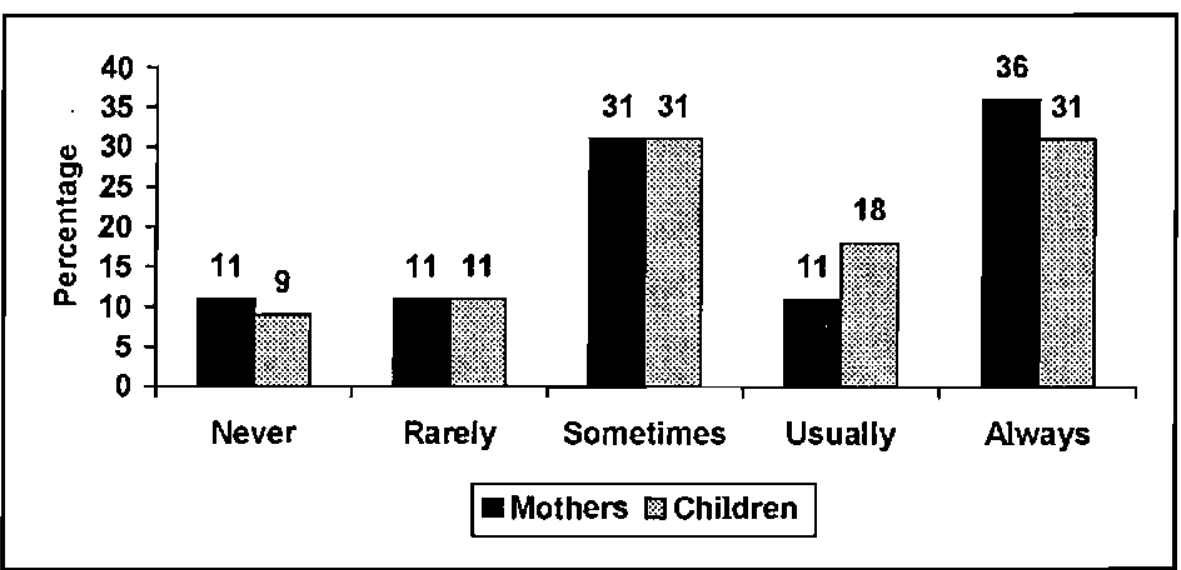

FIGURE 3: Comparison of mothers' and children's use of gestures 
were using drawing to communicate indicates their need to establish effective communication with their mothers. This finding seems to support the reports of many deaf children of the poor communication between themselves and family members. Lane et al. (1996) cites a survey at a school for the deaf which found that only one in ten parents could communicate with his/her deaf child.

In summary it is clear that the three most common methods of communication used in parent-child interaction were speech, signing and gestures. However, the extent to which each was used varied much between both mothers and children as is evident in the means recorded in Table 1. However, children tended to sign more than their mothers. On the other hand, mothers tended to speak more than their children did. This finding has been reported by Bess and Humes (1995) as a general trend with children in signing programmes. The reliance on spoken language as the sole means of communication with a deaf child is said to severely restrict parent-child interaction and interfere with the bonding process and communication with far reaching implications for the child's cognitive, linguistic, emotional and educational progress (Lane et al., 1996).

\section{MOTHERS' DESCRIPTION OF THEIR SIGNING}

Mothers' signing is described in terms of their stated vocabulary, their communication at discourse level, use of sentences and the type of signing used.

\section{Sign vocabulary}

If signing vocabulary by itself was to be used as a yardstick for measuring parents signing skill, the overall results are very low. The majority of mothers $(65 \%)$ had a vocabulary ranging from 0 to 20 words. A further $20 \%$ had a vocabulary of between 21 to 50 words. In effect, $85 \%$ of the sample had a vocabulary size below 51 words. It appears that the majority of mothers had a signing vocabulary that was inadequate for fluent communication

TABLE 2: Mothers' estimation of their signing vocabulary

\begin{tabular}{|l|c|c|}
\hline $\begin{array}{l}\text { Size of } \\
\text { vocabulary }\end{array}$ & $\begin{array}{c}\text { Number } \\
(\mathbf{n = 4 5})\end{array}$ & Percentage \\
\hline 0 & 7 & $16 \%$ \\
\hline $1-10$ & 13 & $29 \%$ \\
\hline $11-20$ & 9 & $20 \%$ \\
\hline $21-50$ & 9 & $20 \%$ \\
\hline $51-100$ & 1 & $2 \%$ \\
\hline $101-200$ & 4 & $9 \%$ \\
\hline $201-300$ & 0 & $0 \%$ \\
\hline 301-500 & 1 & $2 \%$ \\
\hline $501-999$ & 0 & $0 \%$ \\
\hline $1000+$ & 1 & $2 \%$ \\
\hline TOTAL & 45 & $100 \%$ \\
\hline
\end{tabular}

through the medium of signing. The 50-word vocabulary is regarded as an indication of normal developmental level for a speaking child of 18 months, while the average preschool child, according to Yoshinaga-Itano as cited in Jones (1995), is said to have a vocabulary of between 3000 and 6000 words. In addition sign language lexicons have been described as ranging from 1000 to 4000 words, which, even so, is considered a gross underestimation of American Sign Language which is considered to be a fully expressive language (Bellugi, 1980). The Signed English sign system has a lexicon of over 3100 words. The inadequacy of the stated vocabulary of most parents is indicated by the fact that the majority (74\%) of those parents who did sign described their signing vocabulary as allowing them to communicate on only a few topics, while none were able to sign on any topic as reflected in Figure 4.

\section{Communication at discourse level}

The ability to construct sentences is required to communicate efficiently at discourse level. However, only $42 \%$ (16) of the 38 mothers who signed could sign sentences. Results are similar to those obtained by Bornstein (1990) who reported low signing skill at sentence level in a study conducted at Maryland School for the Deaf in the USA. Approximately half the parents could sign sentences after six months of lessons. However, if the entire sample of this study were considered it can be said that only $29 \%$, less than a third, of the sample could sign sentences. Of those who could sign sentences, the majority (81\%) only felt competent to interpret for their own child. None felt competent to communicate with deaf adults. Figure 5 reflects parents' rating of their communication at discourse level.

The ability to communicate with Deaf adults is considered to be an indication of a high level of skill and is used as a measure of proficiency in sign learning programmes. As none of the parents could communicate freely with a Deaf adult, it can be assumed that mastery of signing as a mode of communication had not occurred for any of the parents in the sample. It is acknowledged though, that the level of skill is not the only factor influencing proficiency. Sign dialect could also influence whether or not parents feel competent in communicating with deaf adults. However, this factor was not considered significant in the study as parents were expected to be at least in contact with those who shared a similar dialect because of association with the particular school.

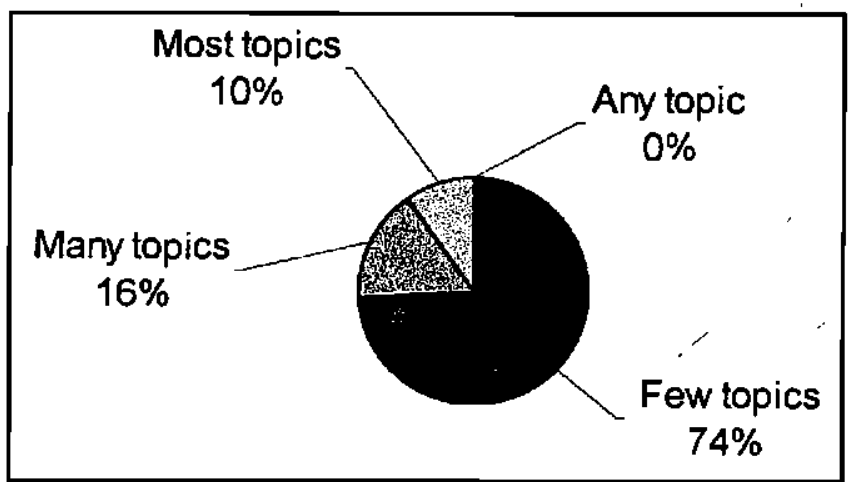

FIGURE 4: Mothers' ability to sign on a range of topics 


\section{Type of signing used}

Home signs constituted the most common type of signing used, with $58 \%$ of signing mothers reporting its use. Two Zulu- speaking mothers who used home signs exclusively were included in this category. One of the mothers reported an elaborate signing system that included a vocabulary of more than 100 signs that allowed her to converse with and interpret for her child. Lane et al. (1996) make reference to this type of communication which develops when a child is unable to communicate with family members through an oral medium and does not have access to a formal signing system. Home signs are said to quickly disappear when children are exposed to formal signing (Stokoe, 1990). It therefore appears that this mother did not have access to the system her child was using at school. The fact that many mothers were using home signs together with formal systems seems to indicate inadequate proficiency in formal signing.

Fingerspelling was the second most common type of signing used, even though it was used by a relatively small percentage of parents, i.e. $38 \%$, who were able to sign the manual alphabet. As fingerspelling supplements signing, it appears that the majority of parents would have difficulty in providing novel information to their children as is accommodated with fingerspelling for those who sign. As fingerspelling is a component of both sign languages and sign systems, parents' lack of this skill could be used to estimate their general signing ability.

On the probe of the description of how they would sign two English sentences which could be taken as a more accurate representation of the type of signing that mothers were using; $44 \%$ (7) of those mothers who could sign at sentence level were using Manually Coded English (MCE). This closely matched the number of mothers who actually stated using this method as reflected in Table 3 . However, only $19 \%$ (3) used key word signing. Thus the other 9 mothers who described themselves as using key word signing (Table 3 ) could not be classified in this category, confirming their lack of knowledge in describing their signing or possibly their poor signing skill. Gregory et al. (1995) also record the tendency of parents to describe themselves as signing despite poor skills, which their

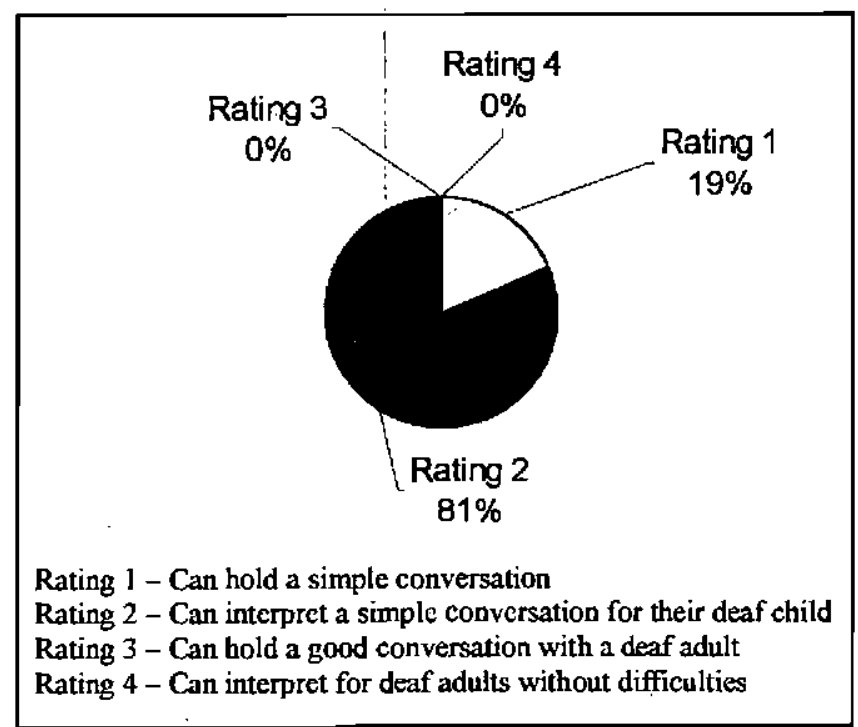

FIGURE 5: Mothers' rating of their communication at discourse level $(n=16)$ young deaf children chose to rather not consider as signing at all when describing their parents' ability to sign. It appears that deaf children used stricter criteria for what constituted signing ability. Only 1 mother $(6 \%)$ appeared to be using sign language. There were 5 mothers (31\%) who could not be classified into a particular system as a result of inconsistencies in their signing. It therefore appears that only 8 of the mothers signed fluently within a prescribed system, that is, $18 \%$ of the sample and $50 \%$ of those mothers who signed at sentence level. Whatever the type of signing used though, it became evident that signing was largely based on English as the vast majority of the 40 mothers who did sign, i.e. $36(90 \%)$, reported speaking and signing at the same time. According to Lane et al. (1996:269), $90 \%$ of teachers surveyed in a 1990 study in the USA reported using simultaneous speech and signing, with over $85 \%$ of teachers using or trying to use MCE. It would therefore appear that parents' responses in this study are similar to those of teachers of the deaf.

It must be noted though, that the use of simultaneous communication has been associated with poor signing skill (Erting, 1980). Furthermore, it has been a contention that simultaneous communication does not serve the purpose of communicative interaction (Sacks, 1989; Lane et al., 1996).

\section{MOTHERS' PERCEPTION OF THEIR SIGNING PROFICIENCY}

A comparison was made between mothers' and children's signing skill to ascertain if mothers' level of signing was in any way influenced by their children's level of skill.

It is clear from their description of their signing proficiency that mothers' signing skill is inadequate in the main. A large percentage of mothers $(29 \%)$ stated that they had no signing skill as such while a further third (33\%) described themselves as having poor skills. Only 5 mothers (11\%) considered themselves better than average. These results are in keeping with the previous description of generally low vocabulary levels and inability to converse at discourse level.

On the probe of their receptive signing ability, mothers rated their receptive signing higher than expressive signing (as depicted in Table 4). This therefore indicates that although mothers described low levels of signing competence, they were able to understand their child's

TABLE 3. Mothers' report of the type of signing used

\begin{tabular}{|l|c|c|}
\hline $\begin{array}{l}\text { Type of } \\
\text { signing used }\end{array}$ & $\begin{array}{c}\text { Number } \\
(\mathbf{n - 4 0})\end{array}$ & Percentage \\
\hline Not sure/unknown & 19 & $48 \%$ \\
Home-signs & 23 & $58 \%$ \\
Fingerspelling & 15 & $38 \%$ \\
Key word signing & 14 & $35 \%$ \\
Manual code of English & 6 & $15 \%$ \\
South African Sign & 3 & $8 \%$ \\
Language & 14 & $35 \%$ \\
Other & \multicolumn{2}{|l}{} \\
\hline
\end{tabular}


signing better than they were able to sign themselves. This seems to indicate that they were realistic in their assessment of their own skills as receptive language is generally judged to be better than expressive language for competent speakers of a language (Bornstein, 1990). Their responses do differ, however, from findings for hearing teachers of the deaf in non-residential, nonintegrated school settings who in the 1985 CADS study (Bornstein, 1990) rated their receptive signing poorer than their expressive signing. It therefore appears that these hearing parents were closer in their responses to teachers of the deaf in residential schools who tended to rate their receptive signing higher, perhaps reflecting a better proficiency and maybe perception of their abilities due to increased contact with signers and exposure to signing. It would appear also that children were signing despite their parents' poor proficiency.

When a comparison of mothers' and children's signing is made, it is evident that mothers considered their children's signing to be superior to their own, as evidenced by the mean score of 3.7 for children, a rating of almost good and 2.2 for mothers, a rating close to poor. It therefore appears that these mothers are generally unable to communicate on an equal level with their children through signing, which is of serious consequence, if signing is the main medium of communication for the child. This could very well be the case as the majority of children in the study $(76 \%)$ were described as either not using spoken language or able to speak on only a few topics. This seems to confirm the impression in the literature that signing children are generally unable to communicate within the family. The study by Gregory et al. (1995) of young Deaf people and their families records only half the number of young people whose preferred language was British Sign Language as having parents who used any type of signing.

To explore possible factors influencing mothers'signing proficiency, relationships with certain demographic details was sought. Results are reflected in Table 5.

It appears that home language was a significant factor. Given the fact that the majority of Zulu-speakers, $27 \%$ of the entire sample, described themselves as not being proficient in English which was the medium of instruction at these schools, it appears that this is impacting on their signing skill. Christensen (1986) highlights the difficulty that monolingual Spanish speaking parents of children attending English medium schools in the USA have in learning to sign. Christensen therefore suggested a trilingual approach as an alternative to bridging the communication gap between English speaking teachers, Spanish speaking parents and signing children. The trilingual approach which consisted of using both the oral languages and conceptual signing was subsequently tried in a two year programme using television as a medium. Results indicated that those parents who viewed the programme regularly improved their signing competence (Christensen, 1986). In addition, positive attitudinal change was noted both towards hearing loss generally as well as toward com- munication with the child.

Thus mothers' signing skill was judged to be inadequate in terms of their own assessment on the rating scale and descriptions of their signing. Only $11 \%$ (5) considered their signing to be good or excellent. The majority of parents had a vocabulary of 50 words or less. As these children are in the first phase of formal schooling and are very much dependent on parent input, the role of parents as the primary educators of their children comes into question. It is evident that the majority of parents in the study are not in a position to support the academic programme as their children are ahead of them in relation to signing skill. As the majority of children were described as having low spoken language ability, it appears that communicative interaction for the majority of mothers and children is inadequate. There is reference in the literature to the signing child growing apart from his family. Gregory et al. - (1995) describe mothers who began learning to sign in order to be part of their growing child's social world. When language barriers between parents and their young child exist, communication is said to become frustrating and stressful as well as superficial (Schoenwald-Oberbeck, 1984). It appears therefore that the subjects in this study are at risk for such a situation.

There have been other indications that the signing skills of parents of deaf children in other regions in South Africa is low, e.g. Lenasia (Cohen, 1996). It therefore appears that sign learning by parents of deaf children is a serious issue especially within this region if not nationally. While schools for the deaf have traditionally carried the responsibility of teaching parents to sign, it appears that a broader response to the issue is needed, involving close co-operation

TABLE 4: Mother's rating of their own and their children's signing skill

(scores on the rating scale were interpreted as: $1=$ no skills, 2 = poor skills, 3 = fair, $4=$ good, $5=$ excellent)

\begin{tabular}{|l|c|c|}
\hline Variable & $\overline{\mathbf{X}}$ & $\overline{\mathbf{S D}}$ \\
\hline Mothers' global singing ability & 2.22 & 1.04 \\
\hline Mother's receptive singing ability & 2.60 & 1.01 \\
\hline Children's signing ability & 3.73 & $\begin{array}{c}0.92 \\
1\end{array}$ \\
\hline
\end{tabular}

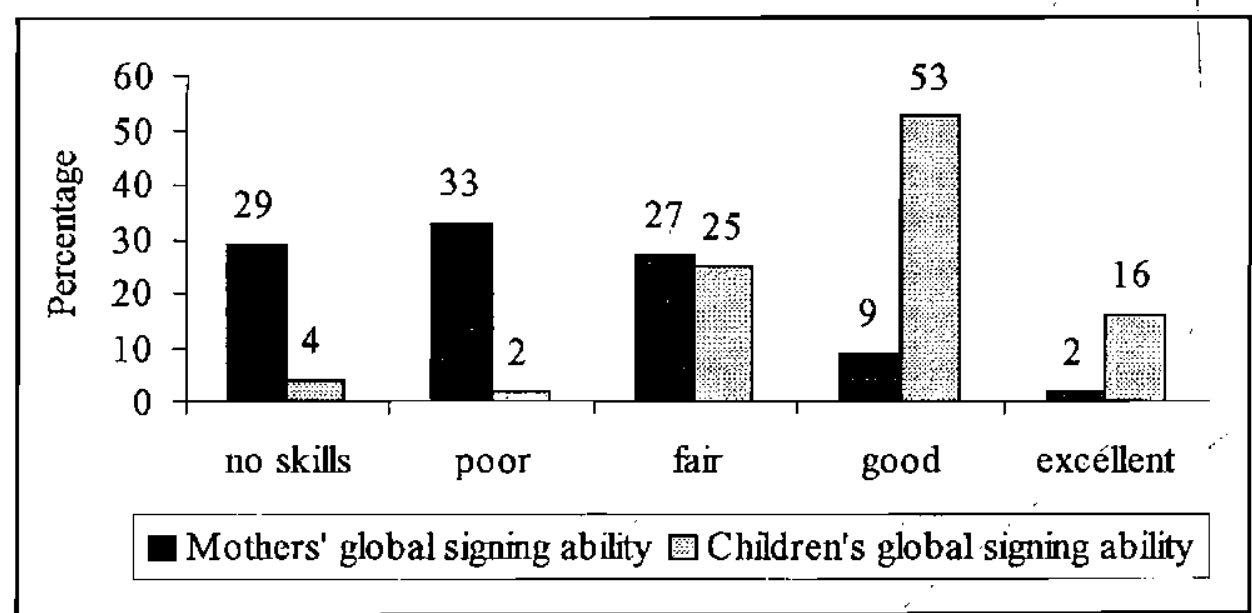

FIGURE 6: Comparison of mothers' and children's signing skill 
between the health and education sectors as well as welfare and non-governmental organisations. Sign learning by hearing parents of deaf children has to be actively promoted at all levels by all professionals working with children who are dependent on a visual mode of communication. Policies and strategies need to be put in place to ensure that opportunities are available, together with the necessary. support for families to learn to communicate through sign language with young deaf members of the family.

\section{CONCLUSION}

The low levels of signing proficiency among hearing mothers of deaf children in signing programmes has implications for both service provision and future research. Fundamental to this would be the stance that parents need to sign and should receive the necessary support when their child is committed to a signing educational programme. As the children in this study were already committed to a methodology, viz. signing, the debates around methodology become less significant in comparison to the issue of parents' communicating meaningfully with their children and supporting the educational programme by becoming competent signers.

Luterman (1987) stresses that professionals recognize that they are working with functional families that could be made more optimal by their intervention. According to McConkey (1985), the implication for all professionals working with helping children is that parental help is necessary if they are to do their jobs efficiently and effectively. He does feel however, that this is "more often preached than practiced" (McConkey, 1985:13). According to Jones (1995) family-professional relationships need to be based on fundamental beliefs that make them workable, such as the belief that families have the capacity to be competent and do have resources. The professional role becomes one of mobilizing and assisting families to draw on their resources. Outcomes are then based on the professional's capacity and desire to promote the family and utilize their resources. The role of the professional therefore is expanded beyond the technical skills of their discipline, in that professionals drive policies and procedures that result in the empowerment of the family.
These principles appear applicable to the audiologist and speech -language therapist who especially in the initial stages, work closely with deaf children and their parents in order to break the communication barrier and prevent isolation of these children within the family.

\section{REFERENCES}

Aron, M. L., Louis, R. E. \& Willemse, J. L. (1986). The issue of signs and the coding of prefix markers by teachers at a school for the deaf. South African Journal of Communication Disorders, 33, 64-72.

Bellugi, U. (1980). How signs express complex meanings. In C. Baker \& R. Batterson. Sign language and the deaf community. USA: National Association for the deaf.

Bess, F. H. \& Humes, L. E. (1995). Audiology: The fundamentals (2nd Ed.) Baltimore: Williams \& Wilkins.

Bornstein, H. (Ed.). (1990). Manual communication: Implications for education. Washington: Gallaudet University Press.

Christensen, K. M. (1986). Conceptual sign language acquisition by Spanish-speaking parents of hearing impaired children. American Annals of the Deaf, 131, 4, 285-287.

Cohen, B. (1996). The development of a sign language resource for parents of deaf children in Lenasia. Unpublished undergraduate research report. Johannesburg: University of the Witwatersrand.

DEAFSA. (1997). Higher education and the needs of the Deaf community: Discussions with the University of South Africa (UNISA). Unpublished paper, Johannesburg.

Erting, C. (1980). Sign language and communication between adults and children. In Baker, C. and Batterson, R. Sign language and the deaf community: Essays in honour of William Stokoe. Silverspring: National Association for the Deaf

Gallaway, C. \& Richards, B. J. (1994). Input and Interaction in language acquisition. New York: Cambridge University Press.

Gregory, S. (1995). Deaf children and their families (2nd Ed). Cambridge: Cambridge University Press.

Gregory, S., Bishop, J. \& Sheldon, L. (1995). Deaf young people and their families: Developing understanding. Cambridge: University Press.

Gregory. S. \& Hartley, G.M. (Eds.). (1991). Constructing deafness. London: Pinter Publishers Limited.

Grove, C. \& Rodda, M. (1984). Receptive communication skills of hearing impaired students: A comparison of four methods of communication. American Annals of the Deaf, 129, 4, 378-385.

Hull, R. H. (1997). Aural rehabilitation: Serving children and adults (3rd Ed). San Diego: Singular Publishing Group.

Jones, A. (1995). Joining the family. Seminars in Hearing, 16, 2 , 196-205.

Joseph, L. (1998). Perceptions of mothers of children in schools for the deaf in Durban with reference to the use of sign language. Unpublished master's dissertation. Pretoria:

TABLE 5: Exploration of selected variables associated with signing proficiency

\begin{tabular}{|c|c|c|c|c|}
\hline Variable & \multicolumn{2}{|c|}{ Mean rank } & p Value & Significance \\
\hline \multirow[t]{2}{*}{ Grade } & Grade 1 & -21.20 & \multirow[t]{2}{*}{.3258} & \multirow[t]{2}{*}{ Not significant } \\
\hline & Grade 3 & -24.89 & & \\
\hline \multirow{2}{*}{$\begin{array}{l}\text { Day versus residential } \\
\text { scholar }\end{array}$} & Day scholar & -23.17 & \multirow[t]{2}{*}{.5996} & \multirow[t]{2}{*}{ Not significant } \\
\hline & Residential & -21.07 & & \\
\hline \multirow[t]{2}{*}{ Home language } & English & -26.57 & \multirow[t]{2}{*}{.0057} & \multirow{2}{*}{$\begin{array}{l}\text { Significant at } \\
<.05 \text { level }\end{array}$} \\
\hline & Zulu & -16.03 & & \\
\hline \multirow[t]{2}{*}{ Gender } & Male & -20.95 & \multirow[t]{2}{*}{.3288} & \multirow[t]{2}{*}{ Not significant } \\
\hline & Female & -24.64 & & \\
\hline
\end{tabular}


University of Pretoria.

Joseph, L. (2000). Bilingual education for deaf children: the challenge to the professions of speech-language pathology and audiology. Paper delivered at the 2 nd International symposium on communication disorders in multilingual populations. 18on communication disorders in multilingual popula

Kyle, J. G. \& Woll, B. (1988). Sign language- the study of deaf people and their language. New York: Cambridge University Press.

Lane, H., Hoffmeister, R. \& Bahan, B. (1996). A journey into the $D E A F$-WORLD. California: DawnSignPress.

Leedy, P. D. (1993). Practical research: Planning and Design. (5th Ed.). New YORK: McMillan Publishing Company.

Luterman, D. (1987). Deafness in the family. Boston: College- hill Press.

Lynas, W. (1994). Communication options in the education of deaf children. London: Whurr Publishers Ltd.

McConkey, R. (1985). Working with parents: A practical guide for teachers and therapists. London: Croomhelm.

Metz, D. E, Caccamise, F. \& Gustafson, M. S. (1997). Criterion validity of the language background questionnaire : A selfassessment instrument. Journal of Communication Disorders, $30,1,23-32$

Mindel, E. D \& Vernon, C. (eds.). (1987). They grow in silence: Understanding Deaf children and adults. Boston: College- Hill Press

National Commission on special needs in education and training (NCSNET) \&' National Committee for education support services (NCESS). (1997). Education for all. From 'Special needs and support' to developing quality education for all learners. Summary of publication document.

Noorbaai, K. (2000). The role of the speech therapist in a school for the deaf. Paper delivered at the 2nd internationa symposium on communication disorders in multilingual populations. 18-21 July. Pilanesberg National Park. South Africa.

Penn, C. (1993). Signs of the times: Deaf language and culture in South Africa. South African Journal of Communication Disorders, 40, 11-23.

Penn, C. \& Reagan, T. G. (1991). Towards a national policy for deaf education in the 'New' South Africa. South African Journal of Communication Disorders, 38, 19-25.

Preston, P. (1994). Mother father deaf : Living between sound and silence. Cambridge: Harvard University Press.

Sacks, O. W. (1989). Seeing voices. A journey into the world of the deaf. Texas: Pro-ed. Inc.

Schoenwald-Oberbeck;'B. (1984). A communication programme for enhancing interaction in families with a hearing impaired child. American Annals of the Deaf, 129, 4, 362-369.

Stokoe, W. C (1990), Language, prelanguage and sign language. Seminars in speech and language, 11, 2, 92-99

Swisher, M. V. \& Thompson, M. (1985). Mothers learning simultaneous communication: The dimensions of the task. American Annals of the Deaf, 130, 3, 212-217.

Woodward, J. (1990). Sign English in education of deaf students. In Bornstein, H. (Ed.). Manual communication: Implications for education. Washington: Gallaudet University Press. 